\title{
Intelligent Interfaces to Empower People with Disabilities
}

\author{
Margrit Betke
}

\section{Introduction}

Severe motion impairments can result from non-progressive disorders, such as cerebral palsy, or degenerative neurological diseases, such as Amyotrophic Lateral Sclerosis (ALS), Multiple Sclerosis (MS), or muscular dystrophy (MD). They can be due to traumatic brain injuries, for example, due to a traffic accident, or to brainstem strokes $[9,84]$. Worldwide, these disorders affect millions of individuals of all races and ethnic backgrounds [4, 75, 52]. Because disease onset of MS and ALS typically occurs in adulthood, afflicted people are usually computer literate. Intelligent interfaces can immensely improve their daily lives by allowing them to communicate and participate in the information society, for example, by browsing the web, posting messages, or emailing friends. However, people with advanced ALS, MS, or MD may reach a point when they cannot control the keyboard and mouse anymore and also cannot rely on automated voice recognition because their speech has become slurred.

People with severe cerebral palsy or traumatic brain injury are often quadriplegic and nonverbal and cannot use the standard keyboard and mouse, or a voice recognition system. In particular children born with severe cerebral palsy can greatly benefit from assistive technology. For them, access to computer technology is empowering. It means access to communication and education - regardless whether their disabilities are purely physical or also cognitive.

To help children and adults with severe disabilities, we initiated the Camera Mouse project several years ago [22]. The project is now a joint effort of researchers at Boston University and Boston College in the United States and has resulted in several camera-based mouse-replacement systems, as well as a system that enables users to create their own gesture-based communication environments. We here

\footnotetext{
Margrit Betke,

Department of Computer Science, Boston University, Boston, MA 02215, USA, e-mail: betke@ c. . bu . edu, www. cs. bu . edu/faculty/betke
} 
Fig. 1 A Camera Mouse user who is quadriplegic due to cerebral palsy. The user moves the mouse pointer to the different regions on the screen by slightly moving his head. In the left upper region of the screen, a window with the Camera Mouse interface can be seen that contains the currently processed video frame showing the user's face. The camera that captured this frame is attached to the top of the monitor (black).

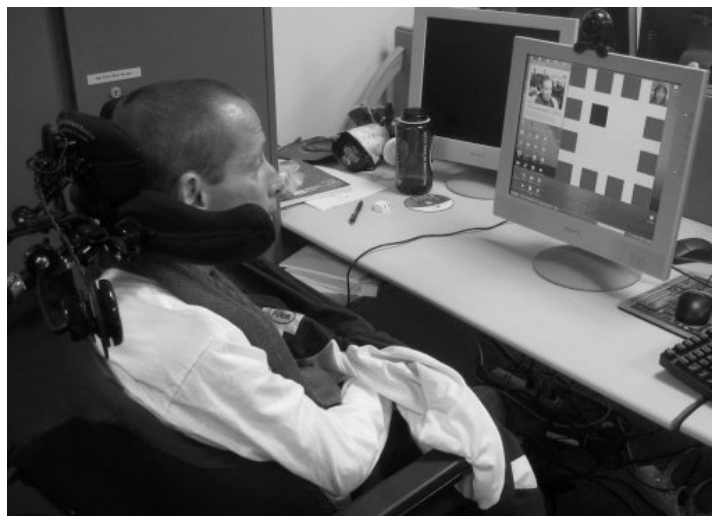

present an overview of these systems (Table 1 and Sections 3, 4 and 5) and the assistive software applications that users can access with these systems (Section 6). Among our interface systems, the generic mouse-replacement system CAMERA MOUSE made the transition from a basic research platform to a popular assistive technology several years ago and is now used worldwide (Figure 1 and Section 2). We also give an overview of smart environments and interface systems for people with disabilities that others have developed (Section 3) and conclude with a discussion of the strengths and limitations of current approaches and the need for future smart environments (Section 7).

Table 1 Camera-based Interface Systems for Users with Severe Motion Impairments

\begin{tabular}{|c|c|}
\hline System & Mecha- Main References \\
\hline Camera Mouse & $\begin{array}{l}\text { Move body part, Betke, Gips, and Fleming [13], } \\
\text { e.g., head, foot, or Betke [10] } \\
\text { finger }\end{array}$ \\
\hline BLINKLINK & $\begin{array}{l}\text { Control blink Grauman, Betke, Lombardi, Gips, } \\
\text { length }\end{array}$ \\
\hline EYEBROW RAISER & Lombardi and Betke [66] \\
\hline EYE KEYS & $\begin{array}{l}\text { Control horizontal Magee, Betke, Gips, Scott, and } \\
\text { gaze direction } \quad \text { Waber [70] }\end{array}$ \\
\hline FINGER COUNTER & Crampton and Betke [27] \\
\hline $\begin{array}{l}\text { HEAD TILT DETEC- } \\
\text { TOR }\end{array}$ & $\begin{array}{l}\text { Tilt head left or Waber, Magee, and Betke [107] } \\
\text { right }\end{array}$ \\
\hline SYMBOLDESIGN & Move body part Betke, Gusyatin, and Urinson [14] \\
\hline
\end{tabular}




\section{The Camera Mouse}

The CAMERA Mouse is used by children and adults of all ages in schools for children with physical or learning disabilities, long-term care facilities for people with advanced neurological diseases, hospitals, and private homes. The CAMERA MOUSE is a camera-based mouse-replacement system that tracks the computer user's movements, typically his or her head or facial movements, and translates them into the movements of the mouse pointer on the screen [13]. To mimic the functionality of a left mouse click, the CAMERA MOUSE evaluates the length of time that the pointer dwells over an icon, button, or menu item (or its surrounding region). If the dwell time is measured to exceed the threshold set by the user, e.g., 0.5 seconds, a selection command is issued.

The Camera Mouse project has worked with various approaches to tracking, e.g., optical flow, template matching with the normalized correlation coefficient as the matching function, and Kalman filtering [13, 33, 26] and with different video cameras [24]. The current version uses optical flow and works with many standard USB video cameras (webcams) from, for example, Logitech, Creative Labs, and Microsoft [22].

Early experiences of users, ages 2-58, working with the CAMERA MOUSE, were described by Gips and Gips [38], Gips, Betke, and DiMattia [39], Cloud, Betke, and Gips [25]. At that time, a couple of dozen systems were in use in the northeastern United States and a school in England. Akram, Tiberii, and Betke [3] explained more recent experiences with a version of the CAMERA MOUSE that calibrates the mapping from feature movement to pointer movement and thus enables users to navigate to all areas of the screen with very limited physical movement.

Our research team has built partnerships with care facilities, such as schools for children with physical or learning disabilities [17] long-term care facilities for people with Multiple Sclerosis and ALS [18], hospitals [23], and private homes [99]. In 2003, 26 schools in Northern Ireland obtained CAMERA MOUSE systems. A breakthrough increase in the number of people who could be helped with the CAMERA MOUSE occurred when our team decided to offer the software for free on the internet. Since it was launched in April 2007, the website www . cameramouse. org has had more than 2,500 downloads a month. The technology's broad impact on the lives of people with disabilities is maybe best explained by testimonials, for example, the words of CAMERA Mouse users from Turkey and Australia (Figure 2).

\section{Intelligent Devices for People with Motion Impairments}

The most basic interface for people with severe motion impairments is a binary switch. This can be a mechanical touch switch, such as a hit plate, a wobble stick, a grip handle, a pinch plate, a sip-and-puff switch, or a pull string. It can also be an electrical switch, such as a voice-activated switch if the person can control making a sound, a tongue-movement detector, or a motion-sensitive photocell or infrared 
Sent: Thursday, July 27, 2006 1:46 AM

Subject: I'm grateful to you

I'm so grateful to you. Because I'm a MS (Multiple Sclerosis) patient since 20 years. I can't move my finger. However I'm in internet fr 10 hour at this time. Thank you very much for everything.

Sincerely

Dr. M. U.

Sent: Thursday, 4 September 2008 16:53:58 +1000

G'day,

[..] a great big thank you from all the way over here in Australia. I have found your fantastic program while looking for an alternative to the expensive trakir and other type head tracking software. [..] I already have a degree of lack of movement in my right arm, shoulder, through to my elbow which also incorporates my ability to grip tightly with my right hand. I am right handed so this is a real pain [..] as well as my condition giving me great pain in movement. I do not take analgesics, I try and keep surfing and swimming and try to keep moving the parts as much as possible. What I am finding is that the program works very well, I am using my daughter's ASUS lap top with built in camera, I can control the cursur OK and feel with more practice will become very proficient. I thank you and your team for your outstanding work in this area.

Best Regards to you all Over there.

L. P.

Fig. 2 Emails from CAMERA Mouse users with motion impairments.

switch. These switches allow users with some motor control to operate wheelchairs or activate selection commands in assistive software [104, 30, 1, 64, 116, 7].

People with cerebral palsy who have some control of their hand movements may be able to use the standard computer mouse as an input device. However, they often have difficulties in positioning the mouse pointer accurately [111, 114]. In an empirical study involving six children with cerebral palsy, Wu and Chen [114] showed that a joystick was a more effective input devices than a trackball or standard mouse. In another study involving four people with muscular dystrophy, Myers et al [73] showed the effectiveness of a stylus-based hand-held personal digital assistant (PDA or Palm) combined with the assistive software Pebbles.

Severe paralysis may leave the eyes as the only muscles that a person can control. Such an individual may be able to control the computer via interfaces that detect eye movements, for example via head-mounted cameras, which typically process infrared signals, [76, 32, 65] or electro-oculography. Systems based on electro-oculography use electrodes placed on the face to detect the movements of the eyes [7, 29, 119]. Eye-movement detection systems are typically more pow- 
erful communication devices than binary switches because they can replace some of the functionality of a standard computer mouse. Another mouse alternative for users with some ability to move their head are infrared head-pointing devices $[31,49,101,93]$.

Many eye trackers require computer users to use head-mounted equipment, for example, glasses with attached infrared transmitters, or to place a reflective "dot" on their foreheads that the infrared systems can track. This is often perceived as intrusive. Electro-oculographic systems can be even more uncomfortable, because electrodes must attached to the user's skin. Users with severe disabilities prefer nonintrusive communication interfaces that passively observe them and do not require any equipment attached to the body. Camera-based systems are therefore particularly suited as intelligent interfaces for people with severe disabilities.

Many current camera-based interfaces make use of active infrared illumination $[50,55,57,72,74,80,87,118]$. Typically, the infrared light reflects off the retina and causes the pupil appear extremely bright in the image, similarly to the "red-eye effect" that occurs in flash photography. The light is synchronized with the camera to illuminate the eyes in alternate frames. The eyes are then located by comparing a frame with this bright pupil effect with a subsequent frame without the illuminated pupils. Typically, the relative gaze direction of an eye is found by analyzing the difference between the center of the bright eye pixel area and the reflection off the surface of the eye from the light source.

Some people are concerned about the high costs of infrared eye trackers, the cognitive challenge posed by such systems, especially for small children or people with physical and cognitive disabilities, and the long-term effect of infrared illumination on the eye. Illuminating the environment instead of the user with infrared light is the strategy of the wearable "gesture pendant," a small wearable camera ringed with infrared light-emitting diodes [37]. The system was designed to control household devices with a hand gesture and may eventually help the elderly or people with disabilities achieve more independence in their homes.

Many other human-computer interaction systems have been proposed that gather data on human motion via video cameras, body suits or gloves, infrared or magnetic markers, head and eye trackers [61]. The focus of the computer vision community has been on the development of methods for detecting and tracking the motion of people without disabilities and analyzing their gestures [86, 54, 103]. These methods, however, may eventually be used to help people with disabilities also. Particularly useful are shape, appearance, and motion models of the human body, in particular, for the head and face [e.g. 117, 63], the hands and fingers [e.g. 108, 6, 45], and the eyes [e.g. 15, 46, 115, 53, 91, 100, 109].

We have focused on smart environments that interpret the user's movements as communication messages, in particular, movements of the user's eyes [70, 89, 12, 11], eyebrows [44, 66], mouth [89], head [107] or fingers [27, 41]. Others have developed camera-based human-computer interaction systems to track the user's nose [e.g. 42], eyes [e.g. 58, 28, 59], or hands [e.g. 19, 113, 36]. 
If users have the ability to make voluntary blinks or winks, they may be assisted by smart environments that can detect intentional eye closures and interpret them as selection commands [e.g. 98, 85, 43, 44].

\section{Camera-Based Music Therapy for People with Motion Impairments}

Moving with music can have an important therapeutic effect on people; playing or creating music while performing movement exercises seems to have an even stronger therapeutic benefit [e.g. 81, 78]. For people with cognitive or severe physical disabilities, however, traditional music instruments may be difficult to play. We therefore developed a smart environment, called MUSIC MAKER, in which people can create music while performing therapeutic exercises [41].

In the MUSIC MAKER environment, a video camera observes the individual's gestures, for example, a reach and grip movement of his or her hand. The system interprets the spatial-temporal characteristics of the movement and provides musical and visual feedback. The user shown in Figure 3, for example, is performing a handopening-and-closing exercise and receives visual feedback on a projection screen. Music is playing during the exercise and is interrupted whenever the user is not opening and closing her fists at a certain minimal speed.

By analyzing the motion pattern and trajectory that a body part follows during an exercise, the MUSIC MAKER environment provides a quantitative tool for monitoring a person's potential recovery process and assessing therapeutic outcomes. In a small empirical study involving subjects without disabilities, we tested the potential of MusicCamera as a rehabilitation tool. The subjects responded to or created music in various movement exercises, such as "keep the music playing," "change the volume of the music" and "play a melody." In these proof-of-concept experiments, the MUSIC MAKER has performed reliably and shown its promise as a therapeutic environment.

Fig. 3 Music MaKeR: A smart environment for music therapy. The user obtains visual and musical feedback about the movement of her hand (Fig. courtesy of Gorman, Lahav, Saltzman, and Betke [41]).

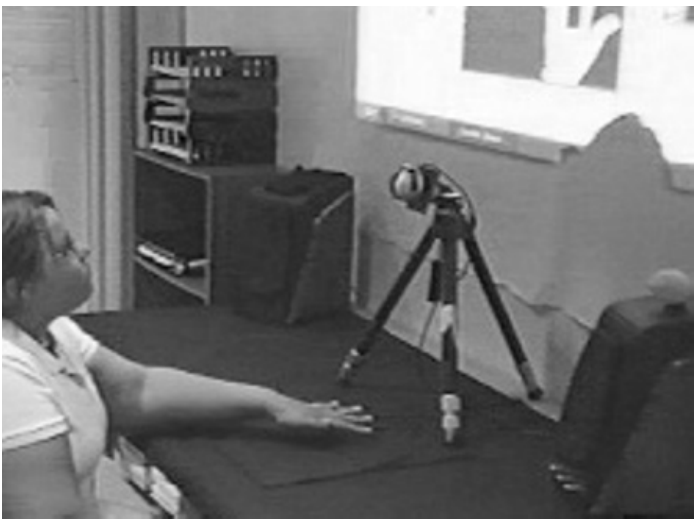




\section{Enabling Users to Create Their Own Smart Environments}

We developed a system, called SYMBOLDESIGN, that allows people to design their own gestures, for example, single-stroke movement patterns they can make by moving their nose tip. With SyMBOLDESIGN, the user can match these gestures to a set of commands that control the computer [14]. For example, the user may decide to choose a zigzag pattern to indicate deletion of a word or a circling gesture to represent the selection command.

SYMBOLDESIGN can be used to design interfaces for pen- or stylus-based input devices [e.g. 71] or for mouse-replacement systems, such as the CAMERA MOUSE. We focused on a user-centered design that is simple and intuitive, tries to minimize user input errors and recognition errors of the system, allows for flexibility, and includes redundancy. Instead of requiring users to memorize and practice predefined symbols, they can create interfaces with SYMBOLDESIGN. This is beneficial because with other symbol or shorthand recognition systems [e.g. 40, 62, 68], the user has to use predefined symbols, whose meaning is often not intuitive and that take time to learn. The ability of SYMBOLDESIGN to process user-defined gestures is particularly important for people with severe motion impairments who can choose gestures based on their movement abilities.

With SymbOLDESIGN, users can create smart environments that "understand" the users' spatio-temporal movement patterns and "learn" how the users typically perform or draw these patterns. The fact that the system does not have a priori information about the alphabet that a person will create makes the pattern classification problem challenging. SYMBOLDESIGN uses a dynamically created classifier, implemented as an artificial neural network, for the machine learning component of the environment. The architecture of the neural network automatically adjusts according to the complexity of the classification task. SYMBOLDESIGN thus creates environments in which the a-priori-unknown number of movement patterns of unknown appearance and structure can be distinguished efficiently.

The issue of gesture similarity arises for anybody who designs a gesture alphabet [67]. An advantage of SyMBOLDESIGN is that users find out during training of the interface if they inadvertently proposed movement patterns that are too similar for the classifier to distinguish reliably. If at least one motion pattern can be reliably recognized, the interface can control assistive software by serving as a binary switch.

\section{Assistive Software for Users with Severe Motion Impairments}

assistive software may be categorized by the level of control of the pointer or cursor on the screen that they require (see Table 2). Some assistive software only works with traditional input devices (mouse, joystick, trackball, stylus) that move the pointer and select items, or with pointer-replacement devices that provide the same functionality (e.g, the CAMERA MOUSE). Other assistive software is specifically designed for alternative input devices that provide less functionality. For example, 
Table 2 Categorization of Assistive Software

\begin{tabular}{|c|c|c|}
\hline Functionality & Input Devices & Application Software \\
\hline $\begin{array}{l}\text { Pointing and } \mathrm{c} \\
\text { ing }\end{array}$ & $\begin{array}{l}\text { Mouse, joystick, } \\
\text { stylus, CAMERA } \\
\text { head tracker }\end{array}$ & $\begin{array}{l}\text { trackball, General software } \\
\text { MousE, }\end{array}$ \\
\hline Pointing only & $\begin{array}{l}\text { Joystick, stylus, } \\
\text { mouse, CAMERA } \\
\text { head tracker }\end{array}$ & $\begin{array}{l}\text { trackball, Stylus-based text entry, e.g., } \\
\text { MousE, using commands defined } \\
\text { with SYMBOLDESIGN, } \\
\text { games, etc. }\end{array}$ \\
\hline Left click only & Binary switches & $\begin{array}{l}\text { Scan-based software, e.g. } \\
\text { text entry, games, etc. }\end{array}$ \\
\hline
\end{tabular}

binary switches can be used to select items in scan-based software, i.e., software that automatically scans through the choices, e.g., letters of the alphabet. Scan-based applications can be created by programming tools called "wifsids," short for "widgets for single-switch input devices" [96] and can adapt to the user's needs by modifying the scan step size and time interval between choices [16].

We engaged in discussions with computer users at various care facilities, for example, The Boston College Campus School [17], which serves students aged 3 to 21 with multiple disabilities, to learn about their most urgent computing needs. We also worked with computer users at The Boston Home [18], a not-for-profit specialized care residence for adults with progressive neurological diseases. Nonverbal people with severe motion impairments desire assistive software that enables them to have access to communication and information, i.e., text entry, email, and web browsing $[10,3]$. They also enjoy playing various computer games [10]. A list of software that we and others have developed for users with severe motion impairments is given in Table 3. Our most popular text entry program is shown in Figure 4. With this software, computer users select letters in a two-step process - first the group of letters and then the desired letter within the group.

The CAMERA Mouse is used regularly by students at The Boston College Campus School [17], e.g., 13 children in Fall 2008. They play with educational games and use the ZAC Browser [120] to access the internet. Some children have cognitive, in addition to severe physical disabilities. For them, the CAMERA MOUSE is a tool to learn the basic principle of cause and effect. They learn that their own physical action (when recognized by the Camera Mouse) can initiate a change in the state of the world (here, the computer software). For these children, a CAMERA MOUSE setup is beneficial that uses a large projection screen to display the child's interaction with application software. The camera mouse control settings are operated by a caregiver on a separate computer monitor and can be changed during the computer session. The caregiver typically selects the tip of the user's nose as the feature to be tracked because the nose is a convenient and robust feature for tracking with the Camera Mouse [25]. 
Table 3 Assistive Software for Users of Our Intelligent Interfaces

\begin{tabular}{|c|c|c|c|}
\hline Assistive Software & Interface System & Task & References \\
\hline NAVIGATETASKS & $\begin{array}{l}\text { CAMERA Mouse, } \\
\text { binary switch }\end{array}$ & $\begin{array}{l}\text { navigation frame- } \\
\text { work (application } \\
\text { mediator) }\end{array}$ & Akram et al [3] \\
\hline MIDAS TOUCH & CAMERa Mouse & text entry & Betke et al [13] \\
\hline $\begin{array}{l}\text { STAGGERED SPEECH, } \\
\text { RICK HOYdT SPELLER }\end{array}$ & CAMERA MOUSE & two-level text entry & $\begin{array}{l}\text { Betke [10], Gips and } \\
\text { Gips [38] }\end{array}$ \\
\hline Dasher & CAMERA MOUSE & $\begin{array}{l}\text { predictive text entry } \\
\text { with language model }\end{array}$ & Ward et al [110] \\
\hline $\begin{array}{l}\text { Web mediator, IWEB- } \\
\text { EXPLORER }\end{array}$ & CAMERA MOUSE & web browsing & $\begin{array}{l}\text { Waber et al [106], Pa- } \\
\text { quette [79] }\end{array}$ \\
\hline ZAC BROWSER & Camera Mouse & web browsing & ZAC Browser [120] \\
\hline $\begin{array}{l}\text { Scan-based on-screen } \\
\text { keyboard }\end{array}$ & BLINKLINK & text entry & Grauman et al [44] \\
\hline ALIENS & Camera Mouse & movement game & Betke et al [13] \\
\hline BLOCK ESCAPE & $\begin{array}{l}\text { EYE KEYS, } \\
\text { CAMERA MOUSE }\end{array}$ & movement game & Magee et al [70] \\
\hline FROG'N FLY & $\begin{array}{l}\text { EYEBROW RAISER, } \\
\text { BLINKLINK }\end{array}$ & movement game & Grauman et al [44] \\
\hline Matching images & $\begin{array}{l}\text { EYEBROW RAISER, } \\
\text { BLINKLINK }\end{array}$ & memory game & Grauman et al [44] \\
\hline PAINT & $\begin{array}{l}\text { Finger COUnter, } \\
\text { CAMERA MOUSE }\end{array}$ & painting & $\begin{array}{l}\text { Crampton and Betke } \\
\text { [27] }\end{array}$ \\
\hline CAMERA CANVAS & Camera Mouse & image editing & Kim et al [60] \\
\hline ANIMATE! & CAMERA Mouse & creating animations & Betke [10] \\
\hline
\end{tabular}

We found that adults who use the CAMERA MOUSE with various application programs like to be able to control the selection of the applications themselves. They prefer to work with the computer as independently from a caregiver as possible. We therefore developed an application mediator system NAVIGATETASKS [2]. It allows CAMERA Mouse users to navigate between different activities such as browsing the internet, posting emails, or listening to music.

For adult computer users at The Boston Home [18], we developed the web browser IWEBEXPLORER [79]. It enables a CAMERA MOUSE user to type a web address via an on-screen keyboard (Figure 5). It also asks the user for confirmation whether he or she indeed wanted to follow a selected link (Figure 5). We added this confirmation mechanism to the browser after noticing that users of standard web browsers often followed links unintentionally. Nearby links were falsely selected because they were so closely located on a web page that they fell within the same area in which the mouse pointer had to dwell to initiate a "left-click" command.

Based on our experience with the IWEBEXPLORER, we developed a web mediator that alters the display of a web page so that the fonts of links become larger 

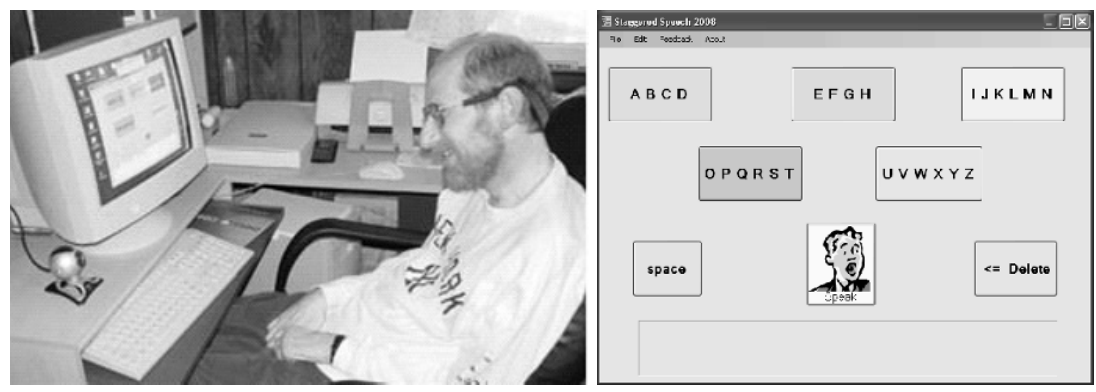

Fig. 4 A user of the two-level text entry program STAGgERED SPEECH. The first level of the on-screen keyboard is shown in both images. The user, a computer scientist who suffered from advanced ALS, took advantage of the CAMERA MOUSE to be on the internet for many hours a day. He composed emails with STAGGERED SPEECH and thereby had a tool to kept in touch with his friends.

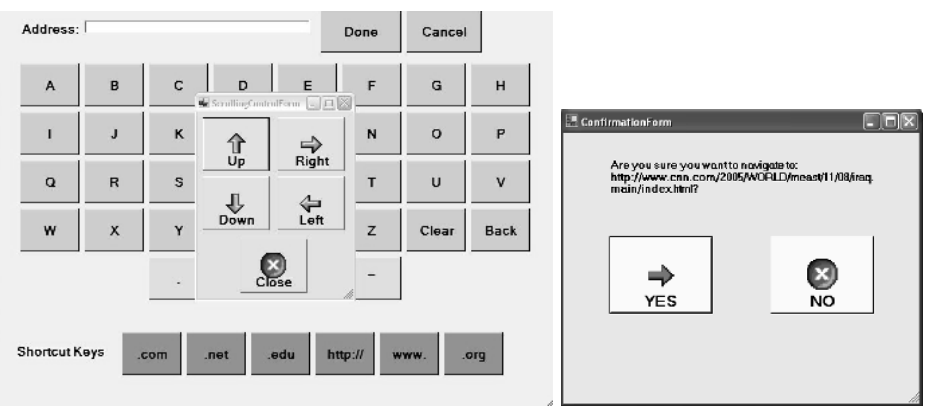

Fig. 5 Assistive windows of the web browser IWEBEXPLORER include an on-screen keyboard with special keys for browsing and a window to confirm the selection of a web link.

[106]. Spatially close web links are grouped to allow users to quickly tab through the groups of links and then the links within these groups. In experiments, we saw a significant reduction in the number of commands needed on average to select a link, which greatly improved the rate of communication. Guidelines for accessible web pages, such as developed by the European project on world-wide augmentative and alternative communication [83], may help encourage web developers to design pages that are easily convertible by web mediators.

For quadriplegic people, being able to dance may be an unrealizable dream. However, although they cannot control the movement of their own hands and feet, they may enjoy using ANIMATE! [5] to control the hands and feet of an anthropomorphic figure. By guiding how the arm, leg, torso, and head segments of the avitar should move from frame to frame, they can, for example, choreograph a dance for the avitar (Figure 6). ANIMATE! thus provides people with severe physical disabilities a tool to explore their creative imagination.

Kim, Kwan, Fedyuk, and Betke [60] recently developed CAMERA CANVAS, an image editing tool for users with severe motion impairments. They studied the solutions for image editing and content creation found in commercial software to design 

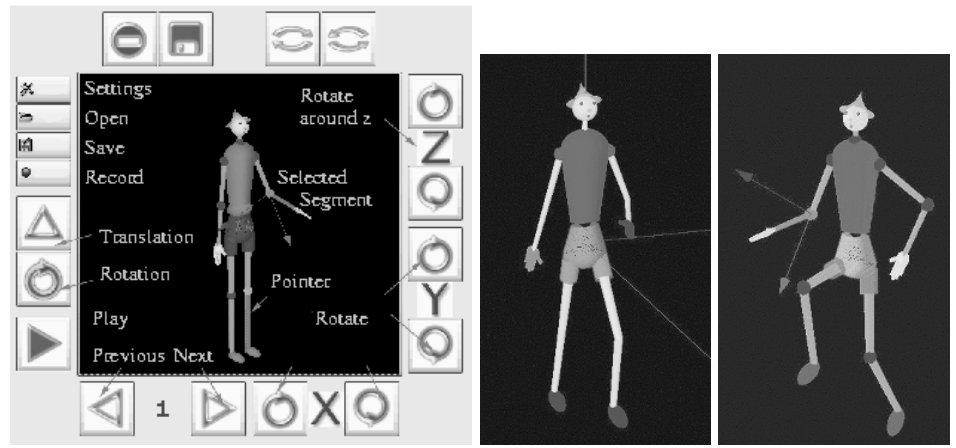

Fig. 6 Animate! is a program that enables people with severe motion impairments to create video animations of an anthropomorphic figure. Its interface has large selection buttons suitable for CAMERA MOUSE use to select commands such as "open a frame," "create a new frame," "save the current frame," "show the previous frame," "show the next frame," "rotate the current frame," and "play all stored frame consecutively." The two image frames shown on the right are part of a video of a dancing figure created with the animation program

a system that provides many of the same functionalities, such as cropping subimages and pasting them into other images, zooming, rotating, adjusting the image brightness or contrast, and drawing with a colored pen, see Fig. 7. Experiments with 20 subjects without disabilities, each testing CAMERA CANVAS with the Camera Mouse as the input mechanism, showed that users found the software easy to understand and operate. In recent experiments with a user with severe cerebral palsy, we found that the he was able to select and enlarge a picture. The user, a young man whose family maintains a website for him, was excited about the potential opportunity to manipulate and create imagery. However, for this user, the procedure to select an item, which involves up or down movement of the menu bar at the left of the graphical interface (see Fig. 7), was too fast and needed to be slowed down. A change of the interface layout, in particular, a horizontal instead of vertical menu bar may also be beneficial for this user, because he was able to control his side-to-side head movements much better than his up-and-down movements.

\section{Discussion}

We have given an overview of the intelligent devices and software applications that we have developed in the past years to empower people with severe disabilities to participate in the information society. Based on our experience in developing these environments and working with people who use them, we suggest that truly multidisciplinary approaches must be taken to build smarter, more powerful environments for people with motion impairments. There are interesting research goals in each of the four disciplines that collectively contribute to the knowledge base of intelligent 


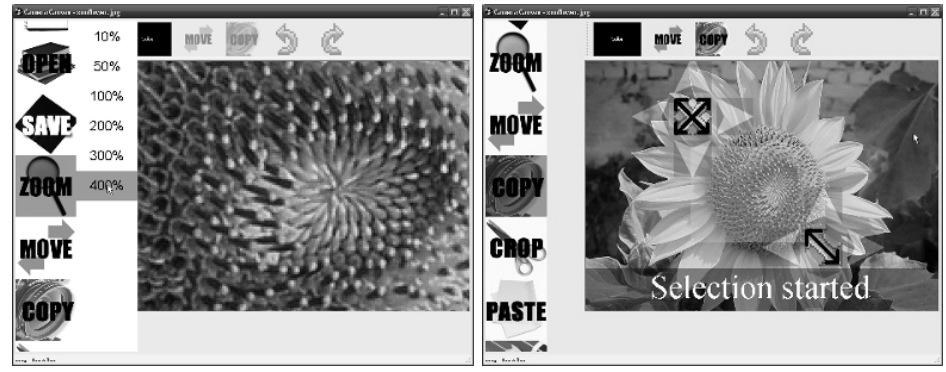

Fig. 7 Image-editing tool Camera Canvas. Left: A Camera Mouse user selected the 400\%zoom option while working with a sunflower picture. Right: To crop and copy an image region, the user must select the position of the upper left corner of the region and the size (i.e., position of lower right corner) of the region by moving the mouse pointer onto one of the arrows.

interface design (Figure 8); significant progress, however, may only be possible if a researcher addressing one task takes into account the impact and dependency of the solutions on the other tasks. We here discuss some of the relevant state-of-the-art approaches within the four disciplines and their strength and limitations.

\section{Computer vision \\ Develop methods to reliably interpret videos of people with disabilities and their voluntary and involuntary movements \\ Human-computer interaction Invent intelligence devices, develop multi- modal interfaces, create viable, potentially user-defined gesture alphabets \\ Assistive software Increase rate of communication of text en- try, improve universal access of the web, create software that automatically adapts to the specific needs of a user \\ Social sciences Bring smart environments to the people who need them - people with disabilities and their caregivers and teachers. Develop methods to teach with smart environments.}

Fig. 8 Disciplines involved in design of intelligent interfaces for people with severe disabilities and main research tasks. 


\subsection{Successful Design of Camera-based Interfaces for People with Disabilities Requires a User-oriented Approach to Computer Vision Research}

The advent of fast processors and inexpensive web cams have contributed to significant advances in computer vision. There has been an explosive growth of the number of camera-based systems that analyze human motion, but an insufficient effort in formal evaluation of these computer-vision systems has been made that is based on user studies [20,82]. Empirical user studies, if conducted at all, sometimes simply recruit subjects from the local population of computer science or engineering students [e.g. 92]. Studies about computer-vision systems that involve users with motion impairments are rare and usually narrow in scope. These studies can be very valuable, because they can bring out the limitations of face trackers, eye detectors, human movement models, etc. when people with motion impairments must rely on them. Magee et al [70], for example, reported that a computer-vision system failed to track the face of a woman with advanced multiple sclerosis, who could not keep her head in an upright position. Another subject had difficulty keeping one eyelid open, which resulted in failure of a gaze-detection system. In scenarios like this, it must be stressed that the source of the problem was not the users' inabilities to conform to the camera-based interface but, conversely, the inability of the interface to adapt to the needs of the users.

Camera-based smart environments that are based on human motion models must be able to automatically interpret the stiff and jerky movements, the involuntary grimacing and tongue thrusting, and the tremors to which some people with severe disabilities are prone and that may increase during periods of emotional stress. We have observed children with spastic cerebral palsy, for example, who became so excited about a computer game that they were playing with the CAMERA MOUSE, that they involuntarily thrust their heads over their shoulders and then could not move for a while.

\subsection{Designing Interfaces for People with Disabilities - Research Efforts in Human-Computer Interaction}

In the area of human-computer interaction, research communities, for example, created by the European Research Consortium for Informatics and Mathematics "User Interfaces for All" (ERCIM UI4All) [UI4All] or the ACM Special Interest Group on Accessible Computing [90], have been developing new assistive devices and methods for universal access to these devices. The new generation of assistive technologies need not involve customized, expensive electro-mechanical devices [21]. Even multi-modal systems need not be expensive, i.e., interfaces involving more than one of a user's senses, typically sight, hearing, and touch, but also smell 
[e.g. 102]. Paradigms have been formulated that provide theoretical frameworks for the principles, assumptions, and practices of accessible and universal design $[95,83]$.

human-centered computing has become an important research area in computer science [77]. It includes efforts to build smart environments that users can control with gestures or symbols [e.g. 113, 37, 36]. Environments that enable users to create their own gesture alphabets have been shown to be particularly appreciated by users $[67,14,97]$.

\subsection{Progress in Assistive Software Development}

Central goals of assistive software development have been to increase the rate of communication for people with disabilities and to improve universal access of the web. Being able to interact with computers to send emails and browse the web can improve the quality of life of these people significantly.

A great advance in increasing the rate of communication of text entry has been made by predictive text-entry systems. While a user is typing, such systems offer word completion based on a language model. Dasher, for example, a wellknown predictive text-entry interface [110], is driven by continuous pointing gestures that can be made with mouse-replacement interfaces such as the CAMERA MousE. Hansen et al [47] developed GAZE TALK, a system that creates dynamic keyboards for several target languages, including Danish, English, German, Italian, and Japanese, and is used with head or eye trackers. Another predictive text entry software is UKO-II by Harbush and Kühn [48]. Letter and word prediction was also used by MacKenzie and Zhang [69] in a system that enabled users to enter text by fixating their eyes on highlighted on-screen keyboard regions. The system detected gaze direction via the head-fixed eye tracker VIEWPOINT [105].

A comparison of the mouse-pointer trajectories of users with and without movement impairments revealed that some users with impairments pause the pointer more often and require up to five times more submovements to complete the same task than users without impairments [51]. Wobbrock and Gajos [111] stressed the difficulty that people with motion impairments have in positioning the mouse pointer within a confined area to execute a click command. An important research focus in the area of assistive software has been the development of strategies to support or alter mouse-pointer movement on the screen. Wobbrock and Gajos [111] suggested that "goal crossing," i.e., selecting something by passing over a target line, is a mechanism that provides higher throughput for people with disability. Bates and Istance [8] created an interface for eye-based pointing devices that increased the size of a target on the screen, such as a button or menu item. Betke, Gusyatin, and Urinson [14] proposed to discretize user-defined pointer-movement gestures in order to extract "pivot points," i.e., screen regions that the pointer travels to and dwells in. A related mechanism are "gravity wells" that draw the mouse pointer into a target on the screen once it is in proximity of the target [e.g. 16]. 
Empirical studies to evaluate quantitatively assistive software that supports or alters mouse-pointer movement on the screen typically measure the time it takes a user to issue a command ("target acquisition") and describe the communication bandwidth using Fitts' law [94, 112, 3]. Qualitative evaluation methods, including interviews with users and caregivers, are also beneficial [3]. We suggest that users perform a cost/benefit analysis when they encounter a new software system. Frankish et al [35], for example, found that for the task of entering a name and phone number into a virtual fax form, users accepted the costs associated with errors of a handwriting recognition system, but for the task of entering a text into a virtual appointment book, they did not. Users may be most inclined to work in smart environments when their physical conditions prevent them from using traditional input devices or when the environments adapt to match their physical abilities.

\subsection{The Importance of Computational Social Science to Intelligent Interface Design}

The study of social implications and applications of computer science has been advocated for decades [e.g. 88]. In recent years, computational social science has emerged as a research field. It may bring about a much-needed bridge between the fields of computer science and the social sciences, in particular, special-needs pedagogy and elderly care. A study by Forrester Research [34] for Microsoft Corporation predicted that the need for accessibility devices would grow in the future due to the increase in computer users above the age of 65 and the increase in the average age of computer users. The study also estimated that about 22.6 million of computer users are currently very likely to benefit from accessible technology. The internet has made it easier for interface developer to inform people with disabilities and their caregivers and teachers about smart environments that they may benefit from. Nonetheless, much effort is still needed to ease the acceptance and use of smart environments in schools, care facilities, and homes, and to develop methods to teach in smart environments.

Research on rehabilitation and disability has lead to a new conceptual foundation for interpreting the phenomenon of disability-a "New Paradigm" of disability [56]. "The 'old' paradigm has presented disability as the result of a deficit in an individual that prevented the individual from performing certain functions or activities." We have a "cultural habit of regarding the condition of the person, not the built environment [..] as the source of the problem." The new paradigm recognizes the contextual aspect of disability - it is "the dynamic interaction between individual and environment over the lifespan that constitutes disability" [56].

A proliferation of smart human-computer environments that can adapt to the physical abilities of every person may help shift our societies' models of disability to the new paradigm. We expect that such environments will cause us to be more inclusive and to accept the notion that physical differences among people are normal. 
Acknowledgements The author would like to thank her collaborators and current and former students who have contributed to the development Camera Mouse and the assistive software described in this paper, in particular, Prof. James Gips at Boston College and Wajeeha Akram, Esra Cansizoglu, Michael Chau, Robyn Cloud, Caitlin Connor, Sam Epstein, Igor Fedyuk, Mikhail Gorman, Oleg Gusyatin, Won-Beom Kim, Chris Kwan, John Magee, Michelle Paquette, Matthew Scott, Maria Shugrina, Laura Tiberii, Mikhail Urinson, Ben Waber, and Emily Yu at Boston University. The author would also like to thank user Rick Hoydt and caregivers Maureen Gates and David Young Hong for their feedback on the Camera Mouse and computer scientist Donald Green for his invaluable implementations.

The published material is based upon work supported by the National Science Foundation under Grant IIS-0713229. Any opinions, findings, and conclusions or recommendations expressed in this material are those of the author and do not necessarily reflect the views of the National Science Foundation.

\section{References}

[1] AbleNet Switches (2008) Roseville, MN, USA. http://www.ablenetinc.com

[2] Akram W, Tiberii L, Betke M (2006) A customizable camera-based human computer interaction system allowing people with disabilities autonomous hands free navigation of multiple computing tasks. In: Stephanidis C, Pieper M (eds) Universal Access in Ambient Intelligence Environments - 9th International ERCIM Workshop "User Interfaces For All" UI4ALL 2006, Königswinter, Germany, September 2006, Revised Papers. LNCS 4397, Springer-Verlag, pp 28-42

[3] Akram W, Tiberii L, Betke M (2008) Designing and evaluating video-based interfaces for users with motion impairments. Universal Access in the Information Society, in review

[4] ALS Association (2008) http: / / www.alsa.org

[5] Animate! (2008) Software for CAmera MOUSE users to create video animations of an anthropomorphic figure. http://csr.bu.edu/ visiongraphics / CameraMouse/animate.html

[6] Athitsos V, Wang J, Sclaroff S, Betke M (2006) Detecting instances of shape classes that exhibit variable structure. In: Computer Vision - ECCV 2006, 9th European Conference on Computer Vision, Graz, Austria, May 7-13, 2006, Proceedings, Part 1, LNCS, Vol. 3951, Springer Verlag, pp 121-134

[7] Barea R, Boquete L, Mazo M, López E (2002) System for assisted mobility using eye movements based on electrooculography. IEEE Transactions on Neural Systems and Rehabilitation Engineering 10(4):209-218

[8] Bates R, Istance H (2002) Zooming interfaces! Enhancing the performance of eye controlled pointing devices. In: Proceedings of the Fifth International ACM Conference on Assistive Technologies (Assets '02), ACM, New York, NY, USA, pp 119-126

[9] Bauby JD (1997) The Diving Bell and the Butterfly. Vintage Books

[10] Betke M (2008) Camera-based interfaces and assistive software for people with severe motion impairments. In: Augusto J, Shapiro D, Aghajan H (eds) 
Proceedings of the 3rd Workshop on "Artificial Intelligence Techniques for Ambient Intelligence” (AITAmI'08), Patras, Greece. 21st-22nd of July 2008. Co-located event of ECAI 2008, Springer-Verlag

[11] Betke M, Kawai J (1999) Gaze detection via self-organizing gray-scale units. In: Proceedings of the International Workshop on Recognition, Analysis, and Tracking of Faces and Gestures in Real-Time Systems, IEEE, Kerkyra, Greece, pp 70-76

[12] Betke M, Mullally WJ, Magee J (2000) Active detection of eye scleras in real time. In: Proceedings of the IEEE Workshop on Human Modeling, Analysis and Synthesis, Hilton Head Island, SC

[13] Betke M, Gips J, Fleming P (2002) The Camera Mouse: Visual tracking of body features to provide computer access for people with severe disabilities. IEEE Transactions on Neural Systems and Rehabilitation Engineering 10(1):1-10

[14] Betke M, Gusyatin O, Urinson M (2006) SymbolDesign: A user-centered method to design pen-based interfaces and extend the functionality of pointer input devices. Universal Access in the Information Society 4(3):223-236

[15] Beymer D, Flickner M (2003) Eye gaze tracking using an active stereo head. In: Proceedings of the 2003 Conference on Computer Vision and Pattern Recognition (CVPR'03) - Volume II, Madison, Wisconsin, pp 451-458

[16] Biswas P, Samanta D (2007) Designing computer interface for physically challenged persons. In: Proceedings of the International Information Technology Conference (ICIT 2007), Rourkella, India, pp 161-166

[17] Boston College Campus School (2008) http://www.bc.edu/ schools / Isoe/campsch

[18] Boston Home (2008) The Boston Home is a specialized care residence for adults with advanced multiple sclerosis and other progressive neurological diseases. http: / / thebostonhome.org

[19] Bouchard B, Roy P, Bouzouane A, Giroux S, Mihailidis A (2008) Towards an extension of the $\mathrm{COACH}$ task guidance system: Activity recognition of Alzheimer's patients. In: Augusto J, Shapiro D, Aghajan H (eds) Proceedings of the 3rd Workshop on "Artificial Intelligence Techniques for Ambient Intelligence” (AITAmI'08), Patras, Greece. 21st-22nd of July 2008. Co-located event of ECAI 2008, Springer-Verlag

[20] Bowyer K, Phillips PJ (1998) Empirical Evaluation Techniques in Computer Vision. IEEE Computer Society Press, Los Alamitos, CA, USA

[21] Brown C (1992) Assistive technology computers and persons with disabilities. Communications of the ACM 35(5):36-45

[22] Camera Mouse (2008) A video-based mouse-replacement interface for people with severe motion impairments. http : / /www. cameramouse.org

[23] Center of Communication Disorders (2008) Children's Hospital, Boston, USA. http://www.childrenshospital.org/ clinicalservices/Site2016/mainpage/S2016P0.html

[24] Chau M, Betke M (2005) Real time eye tracking and blink detection with USB cameras. Tech. Rep. 2005-012, Computer Science Department, 
Boston University, http://www.cs.bu.edu/techreports/pdf/ 2005-012-blink-detection.pdf

[25] Cloud RL, Betke M, Gips J (2002) Experiments with a camera-based humancomputer interface system. In: 7th ERCIM Workshop on User Interfaces for All, Paris, France, pp 103-110

[26] Connor C, Yu E, Magee J, Cansizoglu E, Epstein S, Betke M (2009) Movement and recovery analysis of a mouse-replacement interface for users with severe disabilities. In: Proceedings of the 13th International Conference on Human-Computer Interaction (HCI International 2009), San Diego, CA, in press.

[27] Crampton S, Betke M (2003) Counting fingers in real time: A webcam-based human-computer interface with game applications. In: Proceedings of the Conference on Universal Access in Human-Computer Interaction (UA-HCI), Crete, Greece, pp 1357-1361

[28] Darrell T, Essa IA, Pentland A (1996) Task-specific gesture analysis in real time using interpolated views. IEEE Transactions on Pattern Analysis and Machine Intelligence 18(12):1236-1242

[29] DiMattia P, Curran FX, Gips J (2001) An Eye Control Teaching Device for Students without Language Expressive Capacity - EagleEyes. The Edwin Mellen Press, see also http: / / www . bc . edu / eagleeyes

[30] Don Johnston Switches (2008) Volo, IL, USA. http: / / www . donjohnston. com

[31] Evans DG, Drew R, Blenkhorn P (2000) Controlling mouse pointer position using an infrared head-operated joystick. IEEE Transactions on Rehabilitation Engineering 8(1):107-117

[32] Eye Tracking System, Applied Science Laboratories (2008) Bedford, MA, USA. http: / / www . a-s-1.com

[33] Fagiani C, Betke M, Gips J (2002) Evaluation of tracking methods for humancomputer interaction. In: IEEE Workshop on Applications in Computer Vision, Orlando, Florida, pp 121-126

[34] Forrester Research (2003) The Aging of the US Population and Its Impact on Computer Use. A Research Report commissioned by Microsoft Corporation. http://www.microsoft.com/enable/ research/computerusers.aspx

[35] Frankish C, Hull R, Morgan P (1995) Recognition accuracy and user acceptance of pen interfaces. In: Proceedings of the SIGCHI Conference on Human Factors in Computing Systems (CHI '95), pp 503-510

[36] Freeman WT, Beardsley PA, Kage H, Tanaka K, Kyuman C, Weissman C (2000) Computer vision for computer interaction. SIGGRAPH Computer Graphics 33(4):65-68

[37] Gandy M, Starner T, Auxier J, Ashbrook D (2000) The Gesture Pendant: A self-illuminating, wearable, infrared computer vision system for home automation control and medical monitoring. In: Fourth International Symposium on Wearable Computers (ISWC'00), p 87 
[38] Gips J, Gips J (2000) A computer program based on Rick Hoyt's spelling method for people with profound special needs. In: Proceedings of the International Conference on Computers Helping People with Special Needs (ICCHP), Karlsruhe, Germany, pp 245-250

[39] Gips J, Betke M, DiMattia PA (2001) Early experiences using visual tracking for computer access by people with profound physical disabilities. In: Stephanidis C (ed) Universal Acccess In HCI: Towards an Information Society for All, Volume 3, Proceedings of the 1st International Conference on Universal Access in Human-Computer Interaction (UA-HCI), Lawrence Erlbaum Associates, Mahwah, NJ, pp 914-918

[40] Goldberg D, Richardson C (1993) Touch-typing with a stylus. In: Proceedings of the INTERCHI '93 Conference on Human Factors in Computing Systems, IOS Press, Amsterdam, The Netherlands, pp 80-87

[41] Gorman M, Lahav A, Saltzman E, Betke M (2007) A camera-based music making tool for physical rehabilitation. Computer Music Journal 31(2):39_ 53

[42] Gorodnichy DO, Roth G (2004) Nouse 'use your nose as a mouse' perceptual vision technology for hands-free games and interfaces. Image and Vision Computing 22(12):931-942

[43] Grauman K, Betke M, Gips J, Bradski GR (2001) Communication via eye blinks - detection and duration analysis in real time. In: Proceedings of the IEEE Computer Vision and Pattern Recognition Conference (CVPR), Kauai, Hawaii, vol 2, pp 1010-1017

[44] Grauman K, Betke M, Lombardi J, Gips J, Bradski GR (2003) Communication via eye blinks and eyebrow raises: Video-based human-computer interfaces. International Journal Universal Access in the Information Society 2(4):359-373

[45] Guan H, Chang J, Chen L, Feris R, Turk M (2006) Multi-view appearancebased 3D hand pose estimation. In: IEEE Workshop on Vision for Human Computer Interaction, New York, NY, pp 1-6

[46] Hansen DW, Pece AEC (2005) Eye tracking in the wild. Computer Vision and Image Understanding 98(1):155-181

[47] Hansen JP, Tørning K, Johansen AS, Itoh K, Aoki H (2004) Gaze typing compared with input by head and hand. In: ETRA '04: Proceedings of the 2004 Symposium on Eye Tracking Research \& Applications, ACM, New York, NY, USA, pp 131-138

[48] Harbush K, Kühn M (2003) Towards an adaptive communication aid with text input from ambiguous keyboards. In: 11th Conference of the European Chapter of the Association for Computational Linguistics (EACL 2003)

[49] HeadWay (2008) Infrared head-mounted mouse alternative, Penny \& Giles, Don Johnston, Inc. http: / /www. synapseadaptive.com/ donjohnston/pengild.htm

[50] Hutchinson T, JR KPW, Martin WN, Reichert KC, Frey LA (1989) Humancomputer interaction using eye-gaze input. IEEE Transactions on Systems, Man and Cybernetics 19(6):1527-1533 
[51] Hwang F, Keates S, Langdon P, Clarkson J (2004) Mouse movements of motion-impaired users: a submovement analysis. In: Proceedings of the 6th International ACM SIGACCESS Conference on Computers and Accessibility (Assets '04), pp 102-109

[52] International Myotonic Dystrophy Organization (2008) http://www . myotonic $\backslash$-dystrophy.org

[53] Ivins JP, Porril J (1998) A deformable model of the human iris for measuring small three-dimensional eye movements. Machine Vision and Applications 11(1):42-51

[54] Jaimes A, Sebe N (2005) Multimodal human computer interaction: A survey. In: Sebe N, Lew M, Huang T (eds) Computer Vision in Human-Computer Interaction, ICCV 2005 Workshop on HCI, Beijing, China, October 21, 2005, Proceedings. Lecture Notes in Computer Science, Volume 3766, SpringerVerlag, Berlin Heidelberg, pp 1-15

[55] Ji Q, Zhu Z (2004) Eye and gaze tracking for interactive graphic display. Machine Vision and Applications 15(3):139-148

[56] Kaplan (2008) World Institute on Disability. http://www . accessiblesociety.org

[57] Kapoor A, Picard RW (2002) Real-time, fully automatic upper facial feature tracking. In: Proceedings of the Fifth IEEE International Conference on Automatic Face Gesture Recognition, Washington, D.C., pp 10-15

[58] Kawato S, Tetsutani N (2004) Detection and tracking of eyes for gaze-camera control. Image and Vision Computing 22(12):1031-1038

[59] Kim KN, Ramakrishna RS (1999) Vision-based eye-gaze tracking for human computer interface. In: Proceedings of the IEEE International Conference on Systems, Man, and Cybernetics, Vol. 2, Tokyo, Japan, pp 324-329

[60] Kim WB, Kwan C, Fedyuk I, Betke M (2008) Camera canvas: Image editor for people with severe disabilities. Tech. Rep. 2008-010, Computer Science Department, Boston University, http://www.cs.bu . edu/techreports /pdf / 2008-010-camera-canvas.pdf

[61] Kollios G, Sclaroff S, Betke M (2001) Motion mining: Discovering spatiotemporal patterns in databases of human motion. In: Proceedings of the 2001 ACM SIGMOD Workshop on Research Issues in Data Mining and Knowledge Discovery (DMKD 2001), Santa Barbara, CA, pp 25-32

[62] Kristensson PO, Zhai S (2004) SHARK ${ }^{2}$ : a large vocabulary shorthand writing system for pen-based computers. In: Proceedings of the 17th Annual ACM Symposium on User Interface Software and Technology (UIST '04), ACM, New York, NY, USA, pp 43-52

[63] LaCascia M, Sclaroff S, Athitsos V (2000) Fast, reliable head tracking under varying illumination: An approach based on robust registration of texturemapped 3D models. IEEE Transactions on Pattern Analysis and Machine Intelligence 22(4):322-336

[64] Lankenau A, Röfer T (2000) Smart wheelchairs - state of the art in an emerging market. Künstliche Intelligenz, Schwerpunkt Autonome Mobile Systeme 4:37-39 
[65] LC Technologies Eyegaze System (2008) http : / / www . lctinc . com

[66] Lombardi J, Betke M (2002) A camera-based eyebrow tracker for handsfree computer control via a binary switch. In: 7th ERCIM Workshop on User Interfaces for All, Paris, France, pp 199-200

[67] Long AC, Landay JA, Rowe LA, Michiels J (2000) Visual similarity of pen gestures. In: Proceedings of the SIGCHI Conference on Human Factors in Computing Systems (CHI '00), ACM, New York, NY, USA, pp 360-367

[68] MacKenzie IS, Zhang SX (1997) The immediate usability of graffiti. In: Proceedings of the Conference on Graphics Interface '97, Canadian Information Processing Society, Toronto, Ont., Canada, Canada, pp 129-137

[69] MacKenzie IS, Zhang X (2008) Eye typing using word and letter prediction and a fixation algorithm. In: Proceedings of the 2008 Symposium on Eye Tracking Research \& Applications (ETRA '08), ACM, New York, NY, USA, pp 55-58

[70] Magee JJ, Betke M, Gips J, Scott MR, Waber BN (2008) A human-computer interface using symmetry between eyes to detect gaze direction. Transactions on Systems, Man, and Cybernetics, Part A: Systems and Humans 38(6):1-15

[71] Meyer A (1995) Pen computing: a technology overview and a vision. ACM SIGCHI Bulletin 27(3):46-90

[72] Morimoto CH, Koons D, Amir A, Flickner M (2000) Pupil detection and tracking using multiple light sources. Image and Vision Computing 18(4):331-335

[73] Myers BA, Wobbrock JO, Yang S, Yeung B, Nichols J, Miller R (2002) Using handhelds to help people with motor impairments. In: Proceedings of the Fifth International ACM Conference on Assistive Technologies (Assets '02), ACM, New York, NY, USA, pp 89-96

[74] Myers GA, Sherman KR, Stark L (1991) Eye monitor: microcomputer-based instrument uses an internal mode to track the eye. Computer 24(3):14-21

[75] National Multiple Sclerosis Society (2008) http://www. nationalmssociety.org

[76] Nonaka H (2003) Communication interface with eye-gaze and head gesture using successive DP matching and fuzzy inference. Journal of Intelligent Information Systems 21(2):105-112

[77] NSF HCC (2008) Human-Centered Computing, Division of Information \& Intelligent Systems, National Science Foundation. http : / www. nsf . gov

[78] Pacchetti C, Mancini F, Aglieri R, Fundaro C, Martignoni E, Nappi G (2000) Active music therapy in Parkinson's disease: an integrative method for motor and emotional rehabilitation. Psychosomatic Medicine 62(3):386-393

[79] Paquette M (2005) IWebExplorer, project report, Computer Science Department, Boston University

[80] Park KR (2007) A real-time gaze position estimation method based on a 3-d eye model. IEEE Transactions on Systems, Man, and Cybernetics - Part B: Cybernetics 37(1):199-212 
[81] Paul S, Ramsey D (2000) Music therapy in physical medicine and rehabilitation. Australian Occupational Therapy Journal 47:111-118

[82] Ponweiser W, Vincze M (2007) Task and context aware performance evaluation of computer vision algorithms. In: International Conference on Computer Vision Systems: Vision Systems in the Real World: Adaptation, Learning, Evaluation, Bielefeld, Germany (ICVS 2007)

[83] Poulson D, Nicolle C (2004) Making the internet accessible for people with cognitive and communication impairments. Universal Access in the Information Society 3(1):48-56

[84] Schnabel (2007) Director of the film "The Diving Bell and the Butterfly," France: Pathé Renn Productions

[85] Schwerdt K, Crowley JL (2000) Robust face tracking using color. In: Proceedings of the Fourth IEEE International Conference on Automatic Face and Gesture Recognition, Grenoble, France

[86] Sclaroff S, Betke M, Kollios G, Alon J, Athitsos V, Li R, Magee J, Tian $\mathrm{T}$ (2005) Tracking, analysis, recognition of human gestures in video. In: Proceedings of the 8th International Conference on Document Analysis and Recognition, Seoul, Korea, pp 806-810

[87] Shih SW, Liu J (2004) A novel approach to 3-D gaze tracking using stereo cameras. IEEE Transactions on Systems, Man, and Cybernetics - Part B: Cybernetics 34(1):234-245

[88] Shneiderman B (1971) Computer science education and social relevance. ACM SIGCSE Bulletin 3(1):21-24

[89] Shugrina M, Betke M, Collomosse J (2006) Empathic painting: Interactive stylization through observed emotional state. In: Proceedings of the 4th International Symposium on Non-Photorealistic Animation and Rendering (NPAR 2006), Annecy, France, 8 pp.

[90] SIGACCESS (2008) ACM special interest group on accessible computing. http: / / www.sigaccess.org

[91] Sirohey S, Rosenfeld A, Duric Z (2002) A method of detecting and tracking irises and eyelids in video. Pattern Recognition 35(5):1389-1401

[92] Sirovich L, Kirby M (1987) Low-dimensional procedure for the characterization of human faces. Journal of the Optical Society of America A 4(3):519523

[93] Smart Nav Head Tracker (2008) Natural Point, Eye Control Technologies, Inc., Corvallis, OR, USA. http://www.naturalpoint.com/ smartnav

[94] Soukoreff RW, MacKenzie IS (2004) Towards a standard for pointing device evaluation, perspectives on 27 years of Fitts' law research in HCI. International Journal on Human-Computer Studies 61(6):751-789

[95] Stary C (2006) Special UAIS issue on user-centered interaction paradigms for universal access in the information society. Universal Access in the Information Society 4(3):175-176 
[96] Steriadis CE, Constantinou P (2003) Designing human-computer interfaces for quadriplegic people. ACM Transactions on Computer-Human Interaction 10(2):87-118

[97] StrokeIt (2008) A mouse gesture recognition engine and command processor, software by Jeff Doozan. http: / tcbmi.com/strokeit

[98] Takami O, Morimoto K, Ochiai T, Ishimatsu T (1995) Computer interface to use head and eyeball movement for handicapped people. In: IEEE International Conference on Systems, Man and Cybernetics. Intelligent Systems for the 21st Century, vol 2, pp 1119-1123

[99] Team Hoydt Website (2008) Racing towards inclusion. http://www. teamhoyt.com

[100] Tian Y, Kanade T, Cohn J (2000) Dual-state parametric eye tracking. In: Proceedings of the Fourth IEEE International Conference on Automatic Face and Gesture Recognition, Grenoble, France, pp 110-115

[101] Tracker Pro (2008) Infrared-based head tracker, Madentec, Ltd., Edmonton, Alberta, Canada. http: / /www . madentec. com

[102] Tsugawa S, Aoki M, Hosaka A, Seki K (1994) Recent Japanese projects of AVCS-related systems. In: Proceedings of the Symposium on Intelligent Vehicles, pp 125-130

[103] Turk M (2005) RTV4HCI: A historical overview. In: Kisacanin B, Pavlovic V, Huang T (eds) Real-Time Vision for Human-Computer Interaction, SpringerVerlag

[UI4All] ERCIM working group "User Interfaces for All". http://www. ui4all.gr/index.html

[104] Vaidyanathan R, Chung B, Gupta L, Kook H, Kota S, West JD (2007) Tongue-movement communication and control concept for hands-free human-machine interfaces. IEEE Transactions on Systems, Man, and Cybernetics - Part A: Systems and Humans 37(4):533-546

[105] ViewPoint (2008) Eye tracking system. Arrington Research. http:// www. arring $\backslash$-tonresearch.com

[106] Waber B, Magee JJ, Betke M (2006) Web mediators for accessible browsing. In: Stephanidis C, Pieper M (eds) Universal Access in Ambient Intelligence Environments - 9th International ERCIM Workshop "User Interfaces For All” UI4ALL 2006, Königswinter, Germany, September 2006, Revised Papers. LNCS 4397, Springer-Verlag, pp 447-466

[107] Waber BN, Magee JJ, Betke M (2005) Fast head tilt detection for humancomputer interaction. In: Sebe N, Lew M, Huang T (eds) Computer Vision in Human-Computer Interaction, ICCV 2005 Workshop on HCI, Beijing, China, October 21, 2005, Proceedings. LNCS, Vol. 3766, Springer-Verlag, pp 90-99

[108] Wang J, Athitsos V, Sclaroff S, Betke M (2008) Detecting objects of variable shape structure with hidden state shape models. IEEE Transactions on Pattern Analysis and Machine Intelligence 30(3):477-492

[109] Wang JG, Sung E (2002) Study on eye gaze estimation. IEEE Transactions on Systems, Man, and Cybernetics - Part B: Cybernetics 32(3):332-350 
[110] Ward DJ, Blackwell AF, MacKay DJC (2000) Dasher - a data entry interface using continuous gestures and language models. In: Proceedings UIST 2000: The 13th Annual ACM Symposium on User Interface Software and Technology, http: / / www. inference.phy.cam.ac.uk/dasher

[111] Wobbrock JO, Gajos KZ (2008) Goal crossing with mice and trackballs for people with motor impairments: Performance, submovements, and design directions. ACM Transactions on Accessible Computing 1(1):1-37

[112] Wobbrock JO, Cutrell E, Harada S, MacKenzie IS (2008) An error model for pointing based on Fitts' law. In: Proceeding of the Twenty-sixth Annual SIGCHI Conference on Human Factors in Computing Systems (CHI '08), pp $1613-1622$

[113] Wu C, Aghajan H (2008) Context-aware gesture analysis for speaker HCI. In: Augusto J, Shapiro D, Aghajan H (eds) Proceedings of the 3rd Workshop on “Artificial Intelligence Techniques for Ambient Intelligence" (AITAmI'08), Patras, Greece. 21st-22nd of July 2008. Co-located event of ECAI 2008, Springer-Verlag

[114] Wu TF, Chen MC (2007) Performance of different pointing devices on children with cerebral palsy. In: Stephanidis C (ed) Universal Access in HumanComputer Interaction: Applications and Services, Vol. 4556, Springer-Verlag, Berlin Heidelberg, pp 462-469

[115] Xie X, Sudhakar R, Zhuang H (1995) Real-time eye feature tracking from a video image sequence using Kalman filter. IEEE Transactions on Systems, Man, and Cybernetics 25(12):1568-1577

[116] Yanco HA, Gips J (1998) Driver performance using single switch scanning with a powered wheelchair: Robotic assisted control versus traditional control. In: Proceedings of the Rehabilitation Engineering and Assistive Technology Society of North America Annual Conference (RESNA '98), RESNA Press, pp 298-300

[117] Yang M, Kriegman D, Ahuja N (2002) Detecting faces in images: A survey. IEEE Transactions on Pattern Analysis and Machine Intelligence 24(1):3458

[118] Yoo DH, Chung MJ (2004) Non-intrusive eye gaze estimation without knowledge of eye pose. In: Proceedings of the Sixth IEEE International Conference on Automatic Face and Gesture Recognition, Seoul, Korea, pp 785-790

[119] Young L, Sheena D (1975) Survey of eye movement recording methods. Behavior Research Methods and Instrumentation 7(5):397-429

[120] ZAC Browser (2008) Web browser designed for autistic children. http: / /www.alsa.org 